\title{
MODULARITY IN TOPOLOGICAL LATTICES
}

\author{
DON E. EDMONDSON
}

The purpose of this note is to establish that for topological lattices of suitably small breadth, connectedness implies modularity without an exploitation of compactness. $L$ will denote a topological lattice, that is a Hausdorff topological space with continuous binary operations $\vee$ and $\wedge$ for which $(L, \vee, \wedge)$ is a lattice. For a more explicit presentation and some related machinery, see the paper of E. Dyer and A. Shields [5]. Of specific need, $L$ is a modular lattice iff for every $a, b, c \in L, b \leqq a$ implies $a \wedge(b \bigvee c)=b \bigvee(a \wedge c)$. And if $n$ is a positive integer, the breadth of $L$ is less than $n$ means that for every $x_{1}, x_{2}$, $\cdots, x_{n} \in L$, there exists $j$ such that $x_{j} \leqq \mathrm{~V}_{i \neq j} x_{i}$. Note that the breadth formulation given here is dual to the equivalent phrasing used in [5]. Also for $a \leqq b$ in $L$, define $[a, b]=\{x \in L \mid a \leqq x \leqq b\}$.

THEOREM 1. If $L$ is a connected topological lattice and the breadth of $L$ is less than three, then $L$ is modular.

Proof. It suffices to show that $L$ does not contain a nonmodular five lattice [4]. Thus let $a, b, c \in L$ such that $b \leqq a, a \wedge c=b \wedge c$, and $a \bigvee c=b \bigvee c$. Under the hypotheses of the theorem it will be established that $b=a$, and therefore $L$ cannot contain a nonmodular five lattice.

For $x$ such that $a \leqq x \leqq a \bigvee c$ and $a \wedge c \neq x \wedge c$, let

$$
A=\{y \in[c, c \vee a] \mid a \wedge y \leqq b \vee(x \wedge c)\} .
$$

Clearly $A \cup\{c \vee a\} \subset[c, c \vee a]$. Now if $c \leqq y<c \vee a$, the breadth condition implies (1) $a \wedge y \leqq b \vee(x \wedge c)$, (2) $b \leqq(a \wedge y) \vee(x \wedge c)$, or (3) $x \wedge c \leqq b \bigvee(a \wedge y)$. But (2) implies $b \leqq(a \wedge y) \bigvee c \leqq y$ and hence $a \bigvee c$ $=b \bigvee c \leqq y$, contrary to $y<a \bigvee c$. Also (3) implies $x \wedge c \leqq a$ and therefore $x \wedge c=a \wedge c$, contrary to assumption. Thus if $c \leqq y<c \vee a$, then $y \in A$ and $A \cup\{c \vee a\}=[c, c \vee a]$. But $A$ and $\{c \vee v\}$ are closed nonempty sets whose union is a connected set since $L$ is connected. Thus $c \bigvee a \in A$ and $a=a \wedge(c \bigvee a) \leqq b \bigvee(x \wedge c)$. Therefore $[a, a \bigvee c]=B \cup C$ where

$$
B=\{x \in[a, a \vee c] \mid a \wedge c=x \wedge c\}
$$

and

$$
C=\{x \in[a, a \vee c] \mid a \leqq b \vee(x \wedge c)\}
$$

Received by the editors March 11, 1968. 
Immediately $B$ and $C$ are nonempty closed sets and since $[a, a \bigvee c]$ is connected, $B \cap C \neq \varnothing$. Therefore there exist $d$ such that $a \wedge c=d \wedge c$ and $a \leqq b \vee(d \wedge c)$, and $a \leqq b \vee(d \wedge c)=b \vee(a \wedge c)=b \vee(b \wedge c)=b$. Thus $a=b$.

ThEOREM 2. If $L$ is a locally compact connected topological lattice of codimension less than three, $L$ is modular.

Proof. Lawson [7] has shown that a locally compact connected topological lattice of codimension $n$ has breadth less than or equal to $n$. This generalizes the result of L. W. Anderson and L. E. Ward, Jr. [1] Thus the theorem follows from Theorem 1.

REMARK. In an earlier paper [6] the author gave an example of a compact connected topological lattice of dimension three which is nonmodular. This example is seen to have breadth three also, thus in some respects the result above is the best possible.

\section{REFERENCES}

1. L. W. Anderson and L. E. Ward, Jr., A structure theorem for topological lattices, Proc. Glasgow Math. Assoc. 5 (1961), 1-3.

2. Lee W. Anderson, On breadth and codimension of a topological lattice, Pacific J. Math. 9 (1959), 327-333.

3. - One dimensional topological lattices, Proc. Amer. Math. Soc. 10 (1959), 715-720.

4. Garrett Birkhoff, Lattice theory, Amer. Math. Soc. Colloq. Publ., Vol. 25, Amer. Math. Soc., Providence, R. I., 1948.

5. Eldon Dyer and Allen Shields, Connectivity of topological lattices, Pacific J. Math. 9 (1959), 443-448.

6. Don E. Edmondson, A nonmodular compact connected topological lattice, Proc. Amer. Math. Soc. 7 (1956), 1157-1158.

7. J. D. Lawson, Vietoris mappings and embeddings of topological semi-lattices, Dissertation, Univ. of Tennessee, Knoxville, 1967.

UNIVERSITY OF TEXAS 